\begin{tabular}{ll|l}
\hline Bentham OPEN & The Open AIDS Journal & $\begin{array}{l}\text { The } \\
\text { Open AiDs } \\
\text { lournal }\end{array}$ \\
\hline CrossMark & Content list available at: www.benthamopen.com/TOAIDJ/ & DOI: $10.2174 / 1874613601812010181,2018,12,181-194$ \\
\hline
\end{tabular}

RESEARCH ARTICLE

\title{
Prevalence and Patterns of Antiretroviral Therapy Prescription in the United States
}

\author{
Yunfeng Tie ${ }^{1, *}$, Jacek Skarbinski ${ }^{2}$, Gengsheng Qin ${ }^{3}$ and Emma L Frazier ${ }^{1}$ \\ ${ }^{I}$ Division of HIV/AIDS Prevention, Centers for Disease Control and Prevention, Atlanta, GA, USA \\ ${ }^{2}$ The Permanente Medical Group, Oakland, CA, USA \\ ${ }^{3}$ Department of Mathematics \& Statistics, Georgia State University, Atlanta, GA, USA
}

Received: June 28, 2018

Revised: October 26, 2018

Accepted: October 26, 2018

\begin{abstract}
:
Background:

The use of Antiretroviral Therapy (ART) in HIV-infected persons has proven to be effective in the reduction of risk of disease progression and prevention of HIV transmission.
\end{abstract}

\section{Objective:}

U.S. Antiretroviral Therapy (ART) guidelines specify recommended initial, alternative initial, and not-recommended regimens, but data on ART prescribing practices and real-world effectiveness are sparse.

\section{Methods:}

Nationally representative annual cross sectional survey of HIV-infected adults receiving medical care in the United States, 2009-2012 data cycles. Using data from 18,095 participants, we assessed percentages prescribed ART regimens based on medical record documentation and the associations between ART regimens and viral suppression (most recent viral load test $<200 \mathrm{copies} / \mathrm{ml}$ in past year) and ART-related side effects.

\section{Results:}

Among HIV-infected adults receiving medical care in the United States, 91.8\% were prescribed ART; median time since ART initiation to interview date was 9.8 years. The percentage prescribed ART was significantly higher in 2012 compared to 2009 ( $92.7 \%$ vs $88.7 \% ; p<0.001)$. Of those prescribed ART, $51.6 \%$ were prescribed recommended initial regimens, $6.1 \%$ alternative initial regimens, $29.0 \%$ not-recommended as initial regimens, and $13.4 \%$ other regimens. Overall, $79.5 \%$ achieved viral suppression and $15.7 \%$ reported side effects. Of those prescribed ART and initiated ART in the past year, $80.5 \%$ were prescribed recommended initial regimens.

\section{Conclusion:}

Among persons prescribed ART, the majority were prescribed recommended initial regimens. Monitoring of ART use should be continued to provide ongoing assessments of ART effectiveness and tolerability in the United States.

Keywords: HIV, ART Prescription, ARV regimens, ART initiation, CD4, FDA.

\section{INTRODUCTION}

Antiretroviral Therapy (ART) is recommended for all HIV-infected persons to reduce the risk of disease progression and prevent HIV transmission [1]. By 2017, the U.S. Food and Drug Administration (FDA) has approved more than 26

* Address correspondence to this author at the Division of HIV/AIDS Prevention, Centers for Disease Control and Prevention, 1600 Clifton Road NE, Mailstop E-46, Atlanta, Georgia 30329, Tel: 404-718-5781, Fax no: 404- 639-8640, Email:ytie@cdc.gov 
Individual Antiretroviral (ARV) medications in seven mechanistic classes and several fixed-dose combinations to treat HIV infection [2]. To help clinicians select efficacious, safe and tolerable regimens, the U.S. Department of Health and Human Services (DHHS) guidelines specify three categories of initial ARV regimens for ART-naïve adult patients: recommended, alternative, and not-recommended [1]. As of December 2014, the ten recommended initial regimens have optimal and durable efficacy, favorable tolerability and toxicity profiles, and ease of use. The nine alternative regimens are effective and tolerable but have potential disadvantages when compared with recommended initial regimens. Lastly, 17 regimens are listed that include ARVs not-recommended as initial therapy due to concerns about efficacy, safety or tolerability.

While guideline-specified ARV regimens have been well-studied in research settings in ART-naïve populations [3 9], little is known about HIV providers' prescribing practices and the real-world effectiveness of these regimens in the United States. In practice, ART prescribing and effectiveness could be different from that seen in clinical trials or cohort studies, as ARVs may be used in different populations and settings, and for longer periods of time. We used data from the Medical Monitoring Project (MMP), a nationally representative sample of HIV-infected adults receiving medical care in the United States to obtain population-based estimates of ART prescribing patterns, including the overall percentage of patients prescribed guideline-specified ART as well as specific ARV medications and regimens.

\section{MATERIALS AND METHODS}

\subsection{MMP Study Design}

MMP is an HIV surveillance system that uses an annual, complex-sample, cross-sectional design to produce nationally representative estimates of behavioral and clinical characteristics of HIV-infected adults receiving medical care in the United States [10 - 12]. For the 2009-2012 data collection cycles, first U.S. states and territories were sampled, then facilities providing HIV care, and finally adults aged $\geq 18$ years receiving at least one medical care visit in participating facilities between January and April of the data collection year. For state and territory samples, probability of selection was proportionate to AIDS prevalence; for provider samples, probability of selection was proportionate to HIV-infected patient census. Data were collected annually from June 2009 to May 2013 via patient interviews and medical record abstractions.

MMP data cycles (2009-2012) includes 17 states or territories, which contain about $73 \%$ of all persons living with HIV in the United States [13]. The number of eligible facilities sampled in 2009-2012 data cycles ranged from 603 in 2009 to 548 in 2012. Among those sampled, 461 in 2009 to 467 in 2012 facilities participated, resulting in a mean facility-level response rate of $76.5 \%$ for 2009 and $85.2 \%$ in 2012 . Most of the HIV care facilities sampled were private practices $(48 \%)$, followed by hospital-based facilities $(24 \%)$ and community health centers $(15 \%)$. The remainder facilities were clinical research facilities (8\%), state or local health department clinics $(5 \%)$, community-based service organizations (4\%), and Veterans Administration facilities (3\%).

Of nearly 9,400 sampled persons per data cycle, completed interview and linked medical record abstraction data ranged from 4,217 in 2009 to 5,119 in 2012. All sampled states and territories participated in MMP and the mean facility-level and patient-level response rates for matched interview and medical record data were $82 \%$ and $54 \%$, respectively. All data were weighed based on known probabilities of selection at state or territory, facility, and patient levels and adjusted for non-response using predictors of patient-level response including facility size, facility type (public or private), race/ethnicity, time since HIV diagnosis, and age.

\subsection{Primary Study Variables}

\subsubsection{ART prescription and ARV regimen classifications}

Documentation of ART prescription during the year prior to interview was abstracted from the medical records of MMP participants. Visit dates were used to determine the most recent ART prescription preceding the interview date; individual ARVs were combined into specific ARV regimens, which were grouped into DHHS guideline-specified initial regimen categories (based on December 2014 version of guidelines as a reference for comparisons for regimen types): recommended, alternative, and not-recommended as initial regimens [1]. Regimens not on the DHHS list were classified as other. 


\subsubsection{ART initiation}

Self-reported dates of first ART use were provided during interviews. Using these dates, we examined ART initiation in the past year (recent ART initiation), those who initiated ART more than 1 year ago, and those with unknown ART initiation dates.

\subsubsection{Clinical measures}

To further examine ART prescription patterns, additional clinical measures were examined by ART regimens: 1) recent viral suppression defined as the most recent plasma HIV viral load test in the past year documented in the medical record as undetectable or $<200$ copies/ml; 2) durable viral suppression defined as all plasma HIV viral load tests in the past year documented in the medical record as undetectable or $<200$ copies/ml; 3) self-reported dose adherence in the 72 hours prior to the interview; and 4) self-reported ART-related side effects in the 30 days prior to the interview. A patient was considered adherent if they were currently taking ART and if they were adherent to taking all doses (i.e., taking a dose or set of pills/spoonfuls/injections of ART medications as prescribed by a medical provider). If both conditions did not apply, a patient was considered not adherent.

\subsubsection{Covariates}

To explore the associations between ARV prescription and regimens compared to selected variables, we examined several potential correlates, including demographic and behavioral variables obtained via interview and clinical variables obtained via medical record abstraction or interview. Demographic characteristics included age at interview, gender, race/ethnicity, country or territory of birth, educational level, poverty level, homelessness, incarceration, type of health insurance and coverage. Behavioral characteristics included non-injection drug use for non-medical reasons, binge drinking ( $\geq 5$ drinks per day for men, $\geq 4$ drinks per day for women in the past 30 days), and tobacco use. Clinical factors obtained via interview included depression, with severity calculated using an established algorithm [14, 15], time since HIV diagnosis, and time since ART initiation. Acquired immunodeficiency syndrome (AIDS) diagnosis and CD4+ T-lymphocyte cell (CD4) counts were abstracted from medical records. Disease stage was defined according to Centers for Disease Control and Prevention (CDC) surveillance definitions: Stage 1, no AIDS and nadir CD4 count $\geq$ 500 cells/ $\mu \mathrm{L}$ (or CD4\% $\geq 29$ ); Stage 2, no AIDS and nadir CD4 count 200-499 cells/ $\mu$ L (or CD4\% 14-<29); Stage 3, AIDS or nadir CD4 count $0-199$ cells/ $\mu \mathrm{L}$ (or CD4\% $<14$ ) [16].

\subsection{Statistical Analysis}

First, we calculated weighted percentages and 95\% Confidence Intervals (CIs) for all categorical variables. Medians and Interquartile Ranges (IQRs) were computed for continuous variables. We examined the bivariate associations of those who had been prescribed ART compared to those who had not prescribed ART with potential covariates. The Rao-Scott chi-square test [17] was used to test for differences between groups.

Next, we estimated the weighted prevalence of use of each of the ARV regimens. We described the different clinical characteristics among the ARV regimen classifications and estimated the percentages of participants who had recent viral suppression, durable (all measures) suppression, were dose adherent, and had side effects for all persons who were prescribed ART and those who had initiated ART in the past year.

Furthermore, we examined differences in characteristics between participants who had recent ART initiation to those who initiated ART more than a year ago and estimated the prevalence of ARV regimens among participants who had recent ART initiation. A large number of participants (12.4\%) among those were prescribed ART were excluded from the ART initiation analyses due to missing ART entry dates. To assess the impact of excluding participants with missing ART initiation dates, we completed a sensitivity analysis to compare the socio-demographic characteristics of those with and without ART initiation dates.

Statistical analyses were conducted using SAS (version 9.3, SAS Institute, Cary, North Carolina) and SUDDAN (version 11.0.0, RTI International, Research Triangle Park, North Carolina), and accounted for complex sample survey design. Hypothesis testing results with $p$-values $<0.05$ were considered to be statistically significant.

\subsection{Ethics Statement}

In accordance with the federal human subjects protection regulations, MMP was determined to be a non-research, public health surveillance activity used for disease control program or policy purposes [18, 19]. As such, MMP was not 
subject to federal institutional review board review and received approval for its protocol from CDC officials who were not involved [18]. If required locally, participating states or territories and facilities obtained local institutional review board approval.

\section{RESULTS}

\subsection{ARV Prescription And Regimens}

Of 18,905 MMP participants in 2009-2012 data cycles 16,528 (91.1\%) were prescribed ART in the year prior to interview. Of persons prescribed ART, the majority were male, aged 40 years or older, non-Hispanic black or nonHispanic white, lived above the poverty level, and diagnosed with HIV $\geq 10$ years ago (Table 1). Although, 71.6\% of persons had AIDS (Stage 3), almost half (47.7\%) had a geometric mean CD4 count $\geq 500$ cells/ $\mu \mathrm{L}$ in the past 12 months. Of persons prescribed ART, 79.5\% achieved recent viral suppression, 65.5\% achieved durable viral suppression, $84.4 \%$ reported being adherent with all ART doses in the 72 hours prior to interview, and $15.7 \%$ reported being troubled by ARV-related side effects in the 30 days prior to interview. Persons who were not prescribed ART were more likely to have experienced homelessness or incarceration, used non-injection drugs, be binge drinkers, current smokers, or had depression in the past 2 weeks than those who were prescribed ART.

Table 1. Characteristics of HIV-infected adults receiving medical care in the United States, stratified by prescribed antiretroviral therapy (ART) in past 12 months preceding interview, Medical Monitoring Project, $2009-2012$.

\begin{tabular}{|c|c|c|c|c|c|c|c|}
\hline \multirow{2}{*}{$\begin{array}{c}- \\
\text { Characteristics }\end{array}$} & \multicolumn{2}{|r|}{ Total } & \multicolumn{2}{|c|}{ Prescribed ART } & \multicolumn{2}{|c|}{ Not prescribed ART } & \multirow{2}{*}{$p$-value } \\
\hline & $\mathrm{n}^{\mathrm{a}}$ & $\%(\mathrm{CI})^{\mathrm{b}}$ & $\mathrm{n}^{\mathrm{a}}$ & $\%(\mathrm{CI})^{\mathrm{b}}$ & $\mathrm{n}^{\mathrm{a}}$ & $\%(\mathrm{CI})^{\mathrm{b}}$ & \\
\hline Total & 18095 & 100 & 16528 & $91.1(90.5-91.6)$ & 1567 & $8.9(8.4-9.5)$ & \\
\hline Age at interview (in years) & & & & & & & $<0.001$ \\
\hline $18-29$ & 1343 & $7.6(6.9-8.4)$ & 1086 & $6.7(6.0-7.3)$ & 257 & $17.4(14.3-20.6)$ & \\
\hline $30-39$ & 2841 & $16.0(15.3-16.7)$ & 2517 & $15.6(14.8-16.3)$ & 324 & $20.5(18.0-23.0)$ & \\
\hline $40-49$ & 6396 & $35.1(34.2-35.9)$ & 5882 & $35.3(34.5-36.2)$ & 514 & $32.7(29.6-35.7)$ & \\
\hline$\geq 50$ & 7515 & $41.3(40.4-42.2)$ & 7043 & $42.5(41.5-43.4)$ & 472 & $29.4(26.3-32.5)$ & \\
\hline Gender & & & & & & & 0.001 \\
\hline Male & 13060 & $72.4(70.0-74.8)$ & 12001 & \begin{tabular}{|l|}
$72.8(70.5-75.1)$ \\
\end{tabular} & 1059 & $68.3(64.1-72.5)$ & \\
\hline Female & 4780 & $26.2(23.9-28.6)$ & 4294 & $25.8(23.5-28.1)$ & 486 & $30.5(26.5-34.5)$ & \\
\hline Transgender & 249 & $1.4(1.2-1.6)$ & 228 & $1.4(1.2-1.6)$ & 21 & $1.2(0.6-1.7)$ & \\
\hline Race/Ethnicity & & & & & & & $<0.001$ \\
\hline Non-Hispanic black & 7476 & $41.4(34.8-48.0)$ & 6721 & $40.7(34.0-47.4)$ & 755 & $48.1(41.1-55.0)$ & \\
\hline Hispanic or Latino $^{\mathrm{c}}$ & 3890 & $19.4(15.2-23.6)$ & 3579 & $19.4(15.1-23.7)$ & 311 & $19.0(15.5-22.5)$ & \\
\hline Non-Hispanic White & 5894 & $34.5(29.3-39.7)$ & 5475 & $35.1(29.9-40.4)$ & 419 & $27.5(21.4-33.7)$ & \\
\hline Other $^{d}$ & 821 & $4.8(4.1-5.5)$ & 742 & $4.7(4.0-5.4)$ & 79 & $5.4(3.7-7.1)$ & \\
\hline Foreign Born & & & & & & & 0.220 \\
\hline Born in United States & 15696 & $86.6(85.1-88.1)$ & 14324 & \begin{tabular}{|l|}
$86.5(85.0-88.0)$ \\
\end{tabular} & 1372 & $87.4(85.0-89.9)$ & \\
\hline Born Outside United States & 2392 & $13.4(11.9-14.9)$ & 2198 & $13.5(12.0-15.0)$ & 194 & $12.6(10.1-15.0)$ & \\
\hline Education Attainment & & & & & & & 0.350 \\
\hline$<$ High School & 3976 & $21.0(19.2-22.9)$ & 3644 & $21.2(19.2-23.1)$ & 332 & $19.4(17.2-21.6)$ & \\
\hline High School Diploma or Equivalent & 4935 & $27.0(25.4-28.6)$ & 4504 & $26.9(25.3-28.5)$ & 431 & $27.5(25.0-30.1)$ & \\
\hline$>$ High school & 9177 & $52.0(48.8-55.1)$ & 8374 & $51.9(48.7-55.1)$ & 803 & $53.1(49.4-56.8)$ & \\
\hline Poverty Level in P12M & & & & & & & 0.003 \\
\hline Above Poverty Level & 9426 & $53.8(50.8-56.8)$ & 8659 & $54.1(51.1-57.1)$ & 767 & $50.4(46.3-54.5)$ & \\
\hline At or Below Poverty Level & 8059 & $42.7(39.9-45.5)$ & 7330 & $42.5(39.7-45.3)$ & 729 & $44.8(40.9-48.8)$ & \\
\hline Unknown & 610 & $3.5(2.9-4.1)$ & 539 & $3.4(2.8-4.0)$ & 71 & $4.8(3.4-6.1)$ & \\
\hline Homeless in P12M & 1520 & $8.3(7.6-8.9)$ & 1328 & $7.9(7.2-8.6)$ & 192 & $11.6(9.8-13.4)$ & $<0.001$ \\
\hline Incarcerated in P12M & 907 & $5.1(4.7-5.6)$ & 793 & $5.0(4.5-5.4)$ & 114 & $6.8(5.6-8.0)$ & $<0.001$ \\
\hline Type of Health Insurance in P12M & & & & & & & $<0.001$ \\
\hline Any Private Insurance & 5360 & $31.0(27.9-34.2)$ & 4883 & $30.9(27.8-34.1)$ & 477 & $31.9(27.7-36.1)$ & \\
\hline Public Insurance Only & 9246 & $48.9(46.3-51.5)$ & 8572 & $49.6(47.0-52.2)$ & 674 & $41.3(37.5-45.2)$ & \\
\hline Ryan White Coverage or Uninsured & 3070 & $18.1(15.5-20.6)$ & 2694 & $17.4(14.8-20.0)$ & 376 & $24.8(21.1-28.6)$ & \\
\hline Other Coverage, Not Unspecified & 355 & $2.0(1.5-2.5)$ & 329 & $2.0(1.5-2.6)$ & 26 & $1.9(1.3-2.5)$ & \\
\hline
\end{tabular}


(Table 1) contd....

\begin{tabular}{|c|c|c|c|c|c|c|c|}
\hline \multirow{2}{*}{ Characteristics } & \multicolumn{2}{|r|}{ Total } & \multicolumn{2}{|c|}{ Prescribed ART } & \multicolumn{2}{|c|}{ Not prescribed ART } & \multirow{2}{*}{$p$-value } \\
\hline & $\mathbf{n}^{\mathrm{a}}$ & $\%(C I)^{b}$ & $\mathbf{n}^{\mathrm{a}}$ & $\%(C I)^{b}$ & $\mathbf{n}^{\mathrm{a}}$ & $\%(C I)^{b}$ & \\
\hline Any Non-Injection Drug Use & 4651 & $26.1(24.7-27.5)$ & 4116 & $25.3(23.9-26.6)$ & 535 & $34.8(31.7-38.0)$ & $<0.001$ \\
\hline Binge Drinker in Past 30 days & 2863 & $15.7(15.1-16.3)$ & 2544 & $15.3(14.6-15.9)$ & 319 & $19.8(17.8-21.9)$ & $<0.001$ \\
\hline Current Smoker & 7371 & $40.8(39.2-42.4)$ & 6674 & $40.5(38.9-42.1)$ & 697 & $43.9(41.2-46.6)$ & 0.005 \\
\hline Had Depression in Past 2 Weeks & 4063 & $23.0(21.8-24.2)$ & 3664 & $22.7(21.5-23.9)$ & 399 & $25.8(22.7-28.9)$ & 0.025 \\
\hline \multicolumn{8}{|c|}{ Clinical Indicators } \\
\hline Time since HIV diagnosis (in years) & & & & & & & $<0.001$ \\
\hline$\leq 4$ & 3787 & $22.1(21.1-23.1)$ & 3209 & $20.5(19.6-21.5)$ & 578 & $38.4(34.6-42.2)$ & \\
\hline $5-9$ & 3862 & $21.3(20.6-22.0)$ & 3541 & $21.4(20.6-22.1)$ & 321 & $20.1(17.8-22.3)$ & \\
\hline$\geq 10$ & 10436 & $56.6(55.2-58.0)$ & 9771 & $58.1(56.8-59.4)$ & 665 & $41.5(37.6-45.4)$ & \\
\hline Stage of disease $^{f}$ & & & & & & & $<0.001$ \\
\hline Stage 1 (HIV) & 1208 & $7.0(6.5-7.5)$ & 910 & $5.8(5.2-6.3)$ & 298 & $19.9(17.9-21.8)$ & \\
\hline Stage 2 (HIV) & 4325 & $24.4(23.5-25.3)$ & 3685 & $22.7(21.8-23.6)$ & 640 & $42.3(38.3-46.3)$ & \\
\hline Stage 3 (HIV and AIDS) & 12494 & $68.6(67.6-69.6)$ & 11904 & $71.6(70.5-72.6)$ & 590 & $37.9(34.0-41.7)$ & \\
\hline Geometric mean CD4 count $($ cells $/ \mu \mathrm{L})$ in P12M & & & & & & & $<\mathbf{0 . 0 0 1}$ \\
\hline 0-199 & 2112 & $11.9(11.2-12.7)$ & 2039 & $12.5(11.6-13.3)$ & 73 & $5.5(4.2-6.7)$ & \\
\hline $200-349$ & 2884 & $16.5(15.6-17.3)$ & 2751 & $17.0(16.1-17.9)$ & 133 & $10.5(8.4-12.5)$ & \\
\hline $350-499$ & 3952 & $23.1(22.5-23.8)$ & 3606 & $22.8(22.1-23.5)$ & 346 & $26.8(24.2-29.4)$ & \\
\hline $500+$ & 8323 & $48.4(47.3-49.6)$ & 7581 & $47.7(46.5-48.9)$ & 742 & $57.2(54.6-59.9)$ & \\
\hline $\begin{array}{l}\text { Viral suppression: most recent viral load }<200 \text { copies } / \mathrm{mL} \text { or } \\
\text { undetectable }\end{array}$ & 13559 & $74.7(73.4-76.0)$ & 13150 & $79.5(78.3-80.7)$ & 409 & $25.9(22.8-29.0)$ & $<\mathbf{0 . 0 0 1}$ \\
\hline $\begin{array}{l}\text { Durable viral suppression: all viral load in } \mathrm{P} 12 \mathrm{M}<200 \\
\text { copies } / \mathrm{mL} \text { or undetectable }\end{array}$ & 11218 & $61.6(60.1-63.1)$ & 10869 & $65.5(64.0-66.9)$ & 349 & $22.1(19.2-25.1)$ & $<0.001$ \\
\hline Dose adherence in past 3 days $^{\mathrm{g}}$ & 13890 & $84.4(83.4-85.3)$ & 13468 & $84.4(83.5-85.4)$ & 422 & $81.7(78.3-85.1)$ & 0.087 \\
\hline Reported side effects from ART in past 30 days $^{\mathrm{g}}$ & 2537 & $15.9(15.0-16.8)$ & 2433 & $15.7(14.8-16.5)$ & 104 & $21.8(17.2-26.5)$ & $\mathbf{0 . 0 5 2}$ \\
\hline \multicolumn{8}{|l|}{ Time since ART Initiation } \\
\hline$\leq 1$ year ago & 854 & $5.4(4.9-5.8)$ & 854 & $5.4(4.9-5.8)$ & & & \\
\hline$>1$ year ago & 13733 & $82.2(80.9-83.6)$ & 13733 & $82.2(80.9-83.6)$ & & & \\
\hline Unknown/missing & 1941 & $12.4(11.0-13.7)$ & 1941 & $12.4(11.0-13.7)$ & & & \\
\hline Median ART initiation to interview, in years [IQR] & 14587 & $9.6[3.9-15.4]$ & 14587 & $9.6[3.9-15.4]$ & & & \\
\hline Survey year & & & & & & & $<\mathbf{0 . 0 0 1}$ \\
\hline 2009 & 4217 & $23.2(21.1-25.3)$ & 3737 & $22.6(20.5-24.6)$ & 480 & $29.2(25.6-32.9)$ & \\
\hline 2010 & 4474 & $24.3(22.0-26.7)$ & 4077 & $24.1(21.7-26.5)$ & 397 & $26.6(22.8-30.4)$ & \\
\hline 2011 & 4503 & $26.3(24.0-28.6)$ & 4151 & $26.7(24.3-29.0)$ & 352 & $22.7(18.9-26.4)$ & \\
\hline 2012 & 4901 & $26.2(23.9-28.5)$ & 4563 & 26.7 (24.4-28.9) & 338 & $21.5(17.8-25.3)$ & \\
\hline
\end{tabular}

Abbreviations: CD4, CD4+ T-lymphocyte cell; CI=95\% confidence interval (of weighted percentages); P12M, past 12 month; RW, Ryan White HIV/AIDS Program Assistance.

${ }^{\mathrm{a}}$ Numbers are unweighted.

${ }^{\mathrm{b}}$ Percentages are weighted column percentages.

${ }^{\mathrm{c}}$ Hispanics can be of any race.

${ }^{\mathrm{d}}$ Includes: Asians, American Indians, Alaska Natives, Native Hawaiian and Other Pacific Islanders, and multiracial groups.

${ }^{\mathrm{e}}$ Determined using the U.S. Department of Health and Human Services poverty guidelines. https://aspe.hhs.gov/poverty-guidelines

${ }^{\mathrm{f}}$ Stage 1, no acquired immune deficiency syndrome (AIDS) and nadir CD4+ T-lymphocyte count $\geq 500$ cells/ $\mu \mathrm{L}$ (or CD4\% $\geq 29$ ); Stage 2 , no AIDS and nadir CD4 200-499 cells/ $\mu \mathrm{L}$ (or CD4\% 14-<29); Stage 3, AIDS or nadir CD4 0-199 cells/ $\mu \mathrm{L}$ (or CD4\% $<14$ ).

"Percentages within those who self-reported "currently taking ART".

Among all persons prescribed ART, 51.6\% were prescribed recommended initial regimens, $6.1 \%$ alternative initial regimens, $29.0 \%$ were not-recommended as initial regimens, and 13.4\% were classified as other regimens (Table 2 ). Among all persons prescribed ART, efavirenz (EFV)/tenofovir (TDF)/emtricitabine or lamivudine (XTC) was the most commonly prescribed recommended initial regimen $(27.3 \%)$, followed by ritonavir-boosted atazanavir (ATVr)/TDF/XTC (11.4\%), ritonavir-boosted darunavir (DRVr)/TDF/XTC (4.6\%) and raltegravir (RAL)/TDF/XTC (3.5\%). Among the most commonly prescribed Nucleoside Reverse Transcriptase Inhibitor (NRTI) backbone regimens, the most recently prescribed in the past 12 months were TDF/XTC (64.8\%), abacavir (ABC)/XTC (9.5\%) and zidovudine (ZDV)/XTC (5.9\%) (Supplemental Table 1). The most commonly prescribed individual ARVs were TDF (72.5\%), emtricitabine (FTC) (66.0\%), ritonavir (RTV) (44.5\%), EFV (34.0\%), lamivudine (3TC) (23.0\%), ATV 
(21.6\%), ABC (16.4\%), RAL (14.6\%), DRV (12.6\%), lopinavir (LPV) (10.8\%) and ZDV (10.2\%) (Supplemental Table 2). Very few persons were prescribed non-recommended ARVs, such as stavudine $(0.9 \%)$.

Table 2. Most recently prescribed antiretroviral regimens in the past 12 months preceding the interview of HIV-infected adults receiving medical care in the United States, Medical Monitoring Project, 2009-2012.

\begin{tabular}{|c|c|c|c|c|c|c|}
\hline $\begin{array}{l}\text { DHHS Guideline-Specified ARV } \\
\text { Regimen as of December } 2014\end{array}$ & $\mathbf{n}$ & $\begin{array}{c}\text { Prescribed ARV } \\
\text { Regimen Col\% } \\
\text { (CI) } \\
\end{array}$ & $\begin{array}{c}\text { Recent VL } \\
\text { Suppressed Row\% } \\
\text { (CI) } \\
\end{array}$ & $\begin{array}{c}\text { All VL Suppressed } \\
\text { Row\% (CI) }\end{array}$ & $\begin{array}{c}\text { Dose Adherent } \\
\text { Row\% (CI) }\end{array}$ & $\begin{array}{c}\text { Reported Side } \\
\text { Effects Row\% (CI) }\end{array}$ \\
\hline Recommended Initial Regimens & 8428 & $\begin{array}{c}51.6 \\
(49.6-53.5) \\
\end{array}$ & $\begin{array}{c}81.6 \\
(80.5-82.8) \\
\end{array}$ & $\begin{array}{c}66.3 \\
(64.7-67.9) \\
\end{array}$ & $\begin{array}{c}86.3 \\
(85.3-87.4) \\
\end{array}$ & $\begin{array}{c}14.4 \\
(13.3-15.5) \\
\end{array}$ \\
\hline $\mathrm{EFV} / \mathrm{TDF} / \mathrm{XTC}$ & 4370 & $\begin{array}{c}27.3 \\
(26.1-28.4) \\
\end{array}$ & $\begin{array}{c}85.4 \\
(84.2-86.5) \\
\end{array}$ & $\begin{array}{c}72.6 \\
(70.7-74.5) \\
\end{array}$ & $\begin{array}{c}89.5 \\
(88.3-90.6) \\
\end{array}$ & $\begin{array}{c}14.1 \\
(12.9-15.3)\end{array}$ \\
\hline $\mathrm{ATV} r / \mathrm{TDF} / \mathrm{XTC}$ & 1925 & $\begin{array}{c}11.4 \\
(10.6-12.3)\end{array}$ & $\begin{array}{c}75.9 \\
(73.2-78.5)\end{array}$ & $\begin{array}{c}58.4 \\
(55.2-61.7)\end{array}$ & $\begin{array}{c}83.1 \\
(81.2-85.1)\end{array}$ & $\begin{array}{c}14.1 \\
(12.1-16.2)\end{array}$ \\
\hline $\mathrm{DRVr} / \mathrm{TDF} / \mathrm{XTC}$ & 757 & $\begin{array}{c}4.6 \\
(4.0-5.3) \\
\end{array}$ & $\begin{array}{c}73.2 \\
(69.0-77.3) \\
\end{array}$ & $\begin{array}{c}51.3 \\
(46.7-55.8) \\
\end{array}$ & $\begin{array}{c}83.0 \\
(80.0-86.0) \\
\end{array}$ & $\begin{array}{c}16.5 \\
(13.2-19.9) \\
\end{array}$ \\
\hline RAL/TDF/XTC & 588 & $\begin{array}{c}3.5 \\
(3.1-3.9)\end{array}$ & $\begin{array}{c}80.4 \\
(76.7-84.2)\end{array}$ & $\begin{array}{c}60.7 \\
(56.9-64.6)\end{array}$ & $\begin{array}{c}83.8 \\
(79.9-87.8)\end{array}$ & $\begin{array}{c}11.7 \\
(8.9-14.5)\end{array}$ \\
\hline $\mathrm{ATVr} / \mathrm{ABC} / \mathrm{XTC}$ & 330 & $\begin{array}{c}1.9 \\
(1.7-2.1)\end{array}$ & $\begin{array}{c}82.2 \\
(77.8-86.6)\end{array}$ & $\begin{array}{c}69.7 \\
(64.1-75.4)\end{array}$ & $\begin{array}{c}83.8 \\
(79.8-87.9)\end{array}$ & $\begin{array}{c}16.6 \\
(12.6-20.8)\end{array}$ \\
\hline $\mathrm{EFV} / \mathrm{ABC} / \mathrm{XTC}$ & 227 & $\begin{array}{c}1.4 \\
(1.2-1.6) \\
\end{array}$ & $\begin{array}{c}90.4 \\
(86.1-94.7) \\
\end{array}$ & $\begin{array}{c}81.4 \\
(76.0-86.8) \\
\end{array}$ & $\begin{array}{c}91.1 \\
(86.3-95.9) \\
\end{array}$ & $\begin{array}{c}14.8 \\
(10.0-19.5) \\
\end{array}$ \\
\hline EVG/TDF/FTC & 20 & $\begin{array}{c}0.2 \\
(0.1-0.3)\end{array}$ & $\begin{array}{c}79.3 \\
(61.3-97.3)\end{array}$ & $\begin{array}{c}56.6 \\
(31.7-81.5)\end{array}$ & $\begin{array}{c}29.9 \\
(4.9-54.8)\end{array}$ & $n=4^{\#}$ \\
\hline $\mathrm{RPV} / \mathrm{TDF} / \mathrm{XTC}$ & 211 & $\begin{array}{c}1.4 \\
(1.0-1.7) \\
\end{array}$ & $\begin{array}{c}77.5 \\
(71.6-83.4) \\
\end{array}$ & $\begin{array}{c}52.4 \\
(45.1-59.6) \\
\end{array}$ & $\begin{array}{c}72.8 \\
(67.0-78.5) \\
\end{array}$ & $\begin{array}{c}17.8 \\
(13.0-22.7) \\
\end{array}$ \\
\hline Alternative Initial Regimens & 1013 & $\begin{array}{c}6.1 \\
(5.6-6.6) \\
\end{array}$ & $\begin{array}{c}77.3 \\
(74.3-80.3) \\
\end{array}$ & $\begin{array}{c}63.6 \\
(59.6-67.6) \\
\end{array}$ & $\begin{array}{c}83.0 \\
(79.9-86.0) \\
\end{array}$ & $\begin{array}{c}18.3 \\
(15.5-21.1) \\
\end{array}$ \\
\hline $\mathrm{LPVr}+\mathrm{TDF} / \mathrm{XTC}$ & 642 & $\begin{array}{c}3.9 \\
(3.5-4.3)\end{array}$ & $\begin{array}{c}78.3 \\
(74.8-81.8)\end{array}$ & $\begin{array}{c}65.9 \\
(61.1-70.7)\end{array}$ & $\begin{array}{c}84.0 \\
(80.7-87.3)\end{array}$ & $\begin{array}{c}20.3 \\
(16.6-24.0)\end{array}$ \\
\hline $\mathrm{LPVr}+\mathrm{ABC} / \mathrm{XTC}$ & 149 & $\begin{array}{c}0.9 \\
(0.7-1.1)\end{array}$ & $\begin{array}{c}72.9 \\
(63.5-82.4)\end{array}$ & $\begin{array}{c}60.2 \\
(50.4-70.0)\end{array}$ & $\begin{array}{c}82.4 \\
(74.8-90.0)\end{array}$ & $\begin{array}{c}9.3 \\
(4.3-14.4)\end{array}$ \\
\hline $\mathrm{DRVr}+\mathrm{ABC} / \mathrm{XTC}$ & 126 & $\begin{array}{c}0.7 \\
(0.6-0.8) \\
\end{array}$ & $\begin{array}{c}72.1 \\
(63.2-81.1) \\
\end{array}$ & $\begin{array}{c}48.3 \\
(38.8-57.8) \\
\end{array}$ & $\begin{array}{c}78.4 \\
(71.3-85.5) \\
\end{array}$ & $\begin{array}{c}20.0 \\
(9.9-30.1) \\
\end{array}$ \\
\hline $\mathrm{RAL}+\mathrm{ABC} / \mathrm{XTC}$ & 96 & $\begin{array}{c}0.5 \\
(0.4-0.7) \\
\end{array}$ & $\begin{array}{c}83.8 \\
(72.9-94.7) \\
\end{array}$ & $\begin{array}{c}72.6 \\
(62.0-83.1) \\
\end{array}$ & $\begin{array}{c}82.4 \\
(74.7-90.0) \\
\end{array}$ & $\begin{array}{c}17.0 \\
(9.3-24.6)\end{array}$ \\
\hline $\begin{array}{c}\text { NOT Recommended as Initial } \\
\text { Regimens }\end{array}$ & 4796 & $\begin{array}{c}29.0 \\
(27.5-30.5) \\
\end{array}$ & $\begin{array}{c}79.0 \\
(77.4-80.7) \\
\end{array}$ & $\begin{array}{c}67.4 \\
(65.6-69.2) \\
\end{array}$ & $\begin{array}{c}83.4 \\
(82.1-84.8) \\
\end{array}$ & $\begin{array}{c}16.8 \\
(15.6-18.1) \\
\end{array}$ \\
\hline Other regimens & 2291 & $\begin{array}{c}13.4 \\
(12.6-14.1)\end{array}$ & $\begin{array}{c}73.2 \\
(70.7-75.7)\end{array}$ & $\begin{array}{c}58.8 \\
(56.0-61.6)\end{array}$ & $\begin{array}{c}80.0 \\
(78.0-82.0)\end{array}$ & $\begin{array}{c}16.9 \\
(15.0-18.8)\end{array}$ \\
\hline Total & 16528 & 100 & $\begin{array}{c}79.5 \\
(78.3-80.7)\end{array}$ & $\begin{array}{c}65.5 \\
(64.0-66.9)\end{array}$ & $\begin{array}{c}84.4 \\
(83.5-85.4)\end{array}$ & $\begin{array}{c}15.7 \\
(14.8-16.5)\end{array}$ \\
\hline
\end{tabular}

Abbreviations: n, sample size; \%, weighted percent; CI, 95\% confidence interval; VL, HIV viral load; DHHS, U.S. Department of Health and Human Services.

ARV abbreviations: 3TC, lamivudine; ABC, abacavir; APV, amprenavir; ATV, atazanavir; d4T, stavudine; ddC, zalcitabine; ddI, didanosine; DLV, delavirdine; DRV, darunavir; DTG, dolutegravir; EFV, efavirenz; ETV, etravirine; EVG, elvitegravir; FOS-APV, fosamprenavir; FTC, emtricitabine; IDV, indinavir; LPV, lopinavir; MVC, maraviroc; NFV, nelfinavir; NVP, nevirapine; RAL, raltegravir; r, ritonavir-boosted; RPV, rilpivirine; RTV, ritonavir; SQV, saquinavir; T-20, enfuvirtide; TDF, tenofovir; TPV, tipranavir; XTC, lamivudine or emtricitabine; ZDV, zidovudine.

\# Population estimate was not provided because coefficient of variance exceeded $30 \%$.

We assessed the association between ARV regimens with recent viral suppression, durable viral suppression, dose adherence, and self-reported side effects (Table 2, Fig. 1). Among participants prescribed preferred initial regimens, $81.6 \%$ achieved recent viral suppression, $66.3 \%$ achieved durable viral suppression, $86.3 \%$ reported fully dose-adherent in the 72 hours prior to interview, and $14.4 \%$ reported being troubled by ARV-related side effects in the 30 days prior to interview. For top 5 most frequently prescribed ARV regimens, EFV/TDF/XTC, ATVr/TDF/XTC, DRVr/TDF/XTC, $\mathrm{LPVr} / \mathrm{TDF} / \mathrm{XTC}$, and RAL/TDF/XTC, persons prescribed EFV/TDF/XTC had the highest recent viral suppression (85.4\%), durable viral suppression (72.6\%), fully dose adherent (89.5\%), and lowest in reporting side effects (14.1\%). Among the recommended initial regimens, patients prescribed DRVr/TDF/XTC had the lowest recent viral suppression (73.2\%), durable viral suppression (51.3\%), and dose adherence (83.0\%). Of all regimens, 1 in $5(20 \%)$ of patients prescribed LPVr/TDF/XTC or DRVr/ABC/XTC reported experiencing side effects in the past 30 days. 


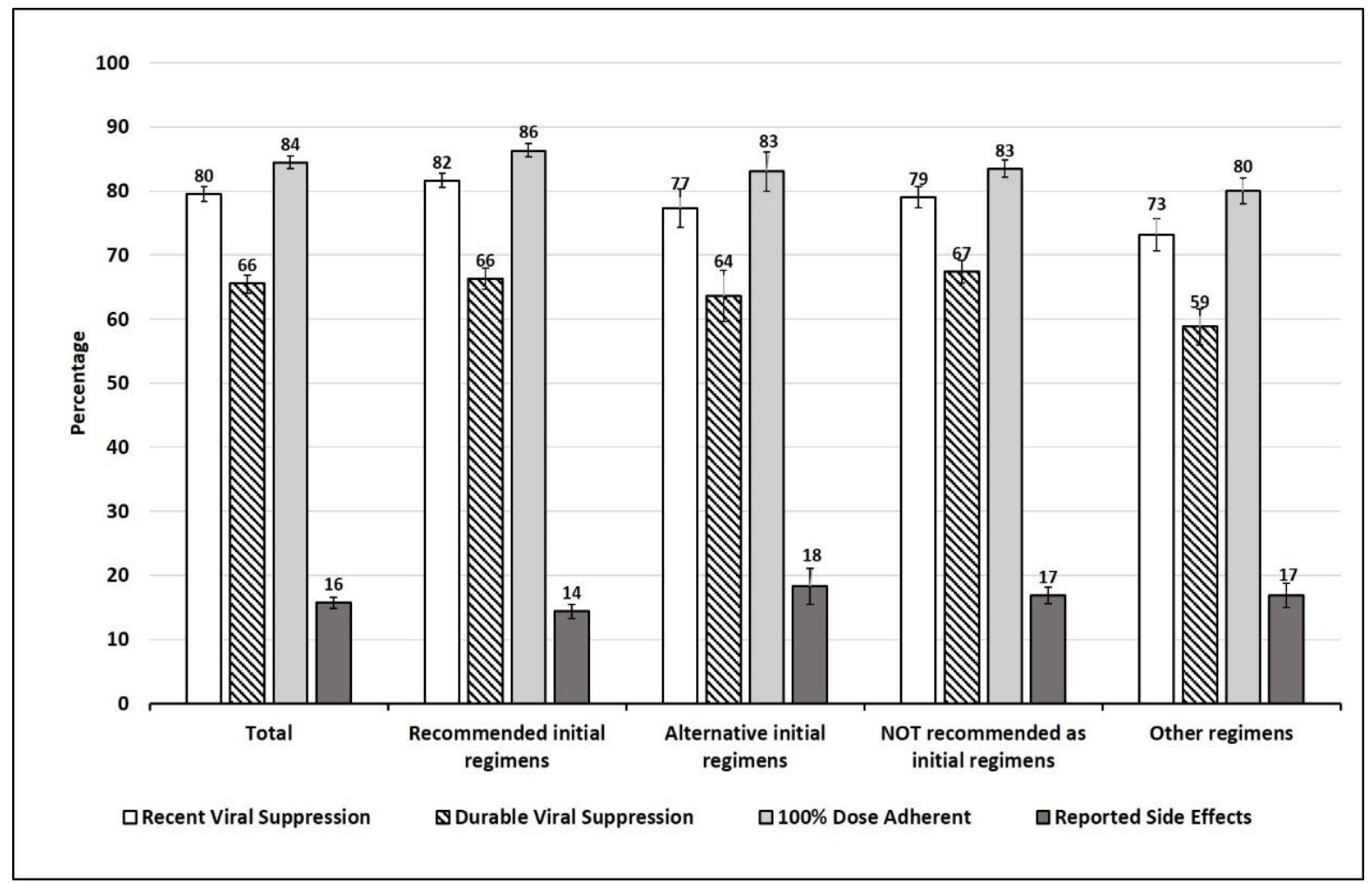

Fig. (1). Prevalence of clinical outcomes among HIV-infected adults receiving medical care that were prescribed antiretroviral regimens, in the past year prior to the interview stratified by type of DHHS guideline-specified ARV regimen, Medical Monitoring Project, 2009-2012.

Recent viral suppression is defined as the most recent viral load in the past 12 months prior to the interview as undetectable or $<200$ copies $/ \mathrm{ml}$. This information is based on data as recorded by medical record abstraction during the past 12 months prior to interview.

Durable viral suppression is defined as all plasma HIV viral load tests in the past year documented in the medical record as undetectable or $<200$ copies $/ \mathrm{ml}$.

ART adherent was defined as patients who self-report that they are currently taking ART and were $100 \%$ dose adherent in the past 3 days. A patient is defined as $100 \%$ adherent if they took their ART doses or set of pills/spoonfuls/injections of ART medications as prescribed by a health care provider in the last 3 days. Otherwise, they were considered as not adherent.

Reported side effects was defined as patients who self-reported that they were currently taking ART and were troubled by side effects from antiretroviral medications in the 30 days prior to the interview.

\subsection{ART Initiation}

Of persons prescribed ART, only $6.1 \%$ were initiated on ART in the past year; median time since ART initiation to interview date was 9.8 years (IQR: 3.9-15.4). About 6 out of $10(6.1 \%)$ participants who reported that they initiated ART in the past year were more likely to be younger ( $<40$ years), non-Hispanic black, at or below poverty level than those who initiated ART more than a year ago (Table 3). About half (47.1\%) of this sample had stage 2 disease and half $(47.3 \%)$ had AIDS (stage 3 ).

Table 3. Characteristics of HIV-infected adults receiving HIV care that were prescribed ART in the United States, stratified by time of ART initiation, Medical Monitoring Project, 2009-2012.

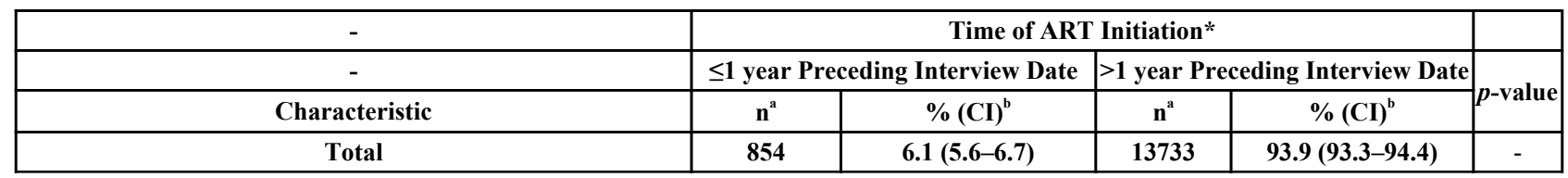




\begin{tabular}{|c|c|c|c|c|c|}
\hline- & \multicolumn{4}{|c|}{ Time of ART Initiation* } & \multirow{3}{*}{$p$-value } \\
\hline- & \multicolumn{2}{|c|}{$\leq 1$ year Preceding Interview Date } & \multicolumn{2}{|c|}{$>1$ year Preceding Interview Date } & \\
\hline Characteristic & $\mathbf{n}^{\mathbf{a}}$ & $\%(C I)^{b}$ & $\mathbf{n}^{\mathbf{a}}$ & $\%(C I)^{b}$ & \\
\hline Age in years & & & & & $<0.001$ \\
\hline $18-29$ & 210 & $24.4(21.2-27.6)$ & 771 & $5.7(5.0-6.3)$ & \\
\hline $30-39$ & 233 & $27.9(24.9-30.9)$ & 2057 & $15.3(14.5-16.1)$ & \\
\hline $40-49$ & 258 & $30.6(26.6-34.7)$ & 4994 & $36.0(35.1-37.0)$ & \\
\hline$\geq 50$ & 153 & $17.1(14.4-19.7)$ & 5911 & $43.0(42.0-44.0)$ & \\
\hline Gender & & & & & 0.111 \\
\hline Male & 636 & $76.1(72.6-79.6)$ & 10006 & $72.9(70.7-75.2)$ & \\
\hline Female & 202 & $22.3(18.8-25.8)$ & 3542 & $25.7(23.5-28.0)$ & \\
\hline Transgender & 14 & $1.6(0.6-2.6)$ & 183 & $1.3(1.1-1.6)$ & \\
\hline Race/Ethnicity & & & & & $<0.001$ \\
\hline Non-Hispanic black & 368 & $43.5(35.8-51.3)$ & 5479 & $39.7(33.2-46.2)$ & \\
\hline Hispanic or Latino $^{c}$ & 205 & $22.1(18.1-26.2)$ & 2908 & $19.2(15.0-23.4)$ & \\
\hline Non-Hispanic white & 233 & $28.1(22.3-34.0)$ & 4722 & $36.5(31.1-41.8)$ & \\
\hline Other $^{d}$ & 48 & $6.2(4.1-8.3)$ & 613 & $4.7(4.0-5.4)$ & \\
\hline Foreign Born & & & & & 0.178 \\
\hline Born in United States & 724 & $84.9(81.7-88.2)$ & 11971 & $86.8(85.3-88.2)$ & \\
\hline Born outside United States & 130 & $15.1(11.8-18.3)$ & 1758 & $13.2(11.8-14.7)$ & \\
\hline Education Attainment & & & & & 0.791 \\
\hline$<$ High school & 166 & $18.9(15.8-22.0)$ & 2783 & $19.5(17.5-21.5)$ & \\
\hline High school diploma or equivalent & 243 & $27.6(23.9-31.4)$ & 3684 & $26.3(24.7-27.9)$ & \\
\hline$>$ High school & 445 & $53.5(48.7-58.2)$ & 7262 & $54.2(50.9-57.4)$ & \\
\hline Poverty level $^{\mathrm{e}}$ in P12M & & & & & 0.041 \\
\hline Above poverty level & 423 & $50.8(46.4-55.2)$ & 7446 & $55.9(52.8-59.1)$ & \\
\hline At or below poverty level & 396 & $45.3(41.0-49.6)$ & 5907 & $41.2(38.3-44.1)$ & \\
\hline Unknown & 35 & $3.9(2.6-5.2)$ & 380 & $2.9(2.3-3.4)$ & \\
\hline Homeless in P12M & 107 & $12.7(10.5-14.8)$ & 1010 & $7.2(6.5-7.9)$ & $<0.001$ \\
\hline Incarcerated in P12M & 58 & $5.9(4.2-7.7)$ & 611 & $4.6(4.1-5.1)$ & 0.136 \\
\hline Type of Health Insurance in P12M & & & & & $<0.001$ \\
\hline Any private insurance & 265 & $32.3(25.6-39.0)$ & 4195 & $32.0(28.8-35.1)$ & \\
\hline Public insurance only & 312 & $32.5(28.1-36.9)$ & 7149 & $49.8(47.1-52.5)$ & \\
\hline Ryan White program coverage only/Uninsured & 245 & $32.1(25.8-38.4)$ & 2092 & $16.2(13.7-18.6)$ & \\
\hline Other coverage (unspecified) & 30 & $3.1(1.3-5.0)$ & 271 & $2.1(1.5-2.6)$ & \\
\hline Any Non-injection Drug Use & 269 & $31.4(27.4-35.5)$ & 3419 & $25.2(23.8-26.6)$ & 0.001 \\
\hline Any Injection Drug Use & 24 & $2.5(1.4-3.6)$ & 322 & $2.2(1.5-2.8)$ & 0.486 \\
\hline Binge Drinker in Past 30 Days & 163 & $20.1(17.2-23.0)$ & 2097 & $15.1(14.4-15.8)$ & $<\mathbf{0 . 0 0 1}$ \\
\hline Current Smoker & 360 & $41.6(37.5-45.7)$ & 5501 & $40.2(38.5-41.8)$ & 0.454 \\
\hline Had depression in past 2 weeks & 223 & $26.5(23.1-30.0)$ & 2963 & $22.0(20.8-23.3)$ & 0.007 \\
\hline \multicolumn{5}{|c|}{ Clinical Indicators } & - \\
\hline Time since HIV diagnosis (in years) & & & & & $<\mathbf{0 . 0 0 1}$ \\
\hline$\leq 4$ & 590 & $71.1(67.4-74.7)$ & 2377 & $18.2(17.2-19.2)$ & - \\
\hline $5-9$ & 140 & $16.3(13.7-18.9)$ & 2990 & $21.7(20.9-22.6)$ & \\
\hline$\geq 10$ & 124 & $12.6(9.9-15.3)$ & 8366 & $60.0(58.6-61.5)$ & \\
\hline Stage of disease ${ }^{f}$ & & & & & $<0.001$ \\
\hline Stage 1 (HIV) & 44 & $5.6(3.5-27.7)$ & 772 & $5.8(5.3-6.4)$ & \\
\hline Stage 2 (HIV) & 380 & $47.1(43.6-50.7)$ & 2908 & $21.3(20.3-22.2)$ & \\
\hline Stage 3 (HIV and AIDS) & 427 & $47.3(43.5-51.0)$ & 10028 & $72.9(71.9-73.9)$ & \\
\hline Geometric mean CD4 count $($ cells/ $\mu \mathrm{L})$ in P12M & & & & & $<0.001$ \\
\hline $0-199$ & 189 & $21.9(18.5-25.2)$ & 1552 & $11.4(10.6-12.3)$ & \\
\hline $200-349$ & 195 & $23.2(20.1-26.3)$ & 2272 & $16.9(16.0-17.9)$ & \\
\hline $350-499$ & 234 & $29.1(26.1-32.2)$ & 2945 & $22.5(21.7-23.3)$ & \\
\hline $500+$ & 218 & $25.8(22.0-29.6)$ & 6487 & $49.1(47.9-50.4)$ & \\
\hline
\end{tabular}


(Table 3) contd....

\begin{tabular}{|c|c|c|c|c|c|}
\hline- & \multicolumn{4}{|c|}{ Time of ART Initiation* } & \multirow{3}{*}{$p$-value } \\
\hline- & \multicolumn{2}{|c|}{$\leq 1$ year Preceding Interview Date } & \multicolumn{2}{|c|}{$>1$ year Preceding Interview Date } & \\
\hline Characteristic & $\mathrm{n}^{\mathrm{a}}$ & $\%(C I)^{b}$ & $\mathrm{n}^{\mathrm{a}}$ & $\%(\mathrm{CI})^{\mathrm{b}}$ & \\
\hline $\begin{array}{l}\text { Viral suppression: most recent viral load }<200 \text { copies } / \mathrm{mL} \text { or } \\
\text { undetectable }\end{array}$ & 592 & $68.6(65.1-72.2)$ & 11100 & $80.6(79.4-81.9)$ & $<\mathbf{0 . 0 0 1}$ \\
\hline $\begin{array}{l}\text { Durable viral suppression: all viral load in P12M }<200 \\
\text { copies } / \mathrm{mL} \text { or undetectable }\end{array}$ & 132 & $14.7(12.0-17.4)$ & 9503 & $68.8(67.3-70.3)$ & $<0.001$ \\
\hline Dose adherence in past 3 days $^{\mathrm{g}}$ & 690 & $83.7(81.4-86.1)$ & 11348 & $85.0(84.0-86.0)$ & 0.280 \\
\hline Reported side effects from ART in past 30 days $^{g}$ & 141 & $17.5(14.2-20.8)$ & 2062 & $15.8(14.9-16.8)$ & 0.338 \\
\hline Survey year & & & & & 0.001 \\
\hline 2009 & 247 & $29.1(24.0-34.3)$ & 3092 & $22.5(20.6-24.5)$ & \\
\hline 2010 & 229 & $26.2(21.3-31.0)$ & 3341 & $23.8(21.7-25.9)$ & \\
\hline 2011 & 191 & $23.1(18.8-27.3)$ & 3448 & $26.6(24.5-28.6)$ & \\
\hline 2012 & 187 & $21.6(17.1-26.1)$ & 3852 & $27.1(24.9-29.4)$ & \\
\hline
\end{tabular}

* Participants with missing ART initiation date were excluded from this Table.

Abbreviations: $\mathrm{CI}=$ confidence interval (of weighted percentages); P12M, past 12 month; RW, Ryan White HIV/AIDS Program Assistance; CD4, CD4+ T-lymphocyte cell.

${ }^{a}$ Numbers are unweighted.

${ }^{\mathrm{b}}$ Percentages are weighted percentages.

${ }^{\mathrm{c}}$ Hispanics can be of any race.

${ }^{\mathrm{d}}$ Includes: Asians, American Indians, Alaska Natives, Native Hawaiian and Other Pacific Islanders, and multiracial groups.

${ }^{\mathrm{e}}$ Determined using the U.S. Department of Health and Human Services poverty guidelines. https://aspe.hhs.gov/poverty-guidelines

${ }^{\mathrm{f}}$ Stage 1, no acquired immune deficiency syndrome (AIDS) and nadir CD4+ T-lymphocyte count $\geq 500$ cells $/ \mu \mathrm{L}$ (or CD4\% $\geq 29$ ); Stage 2 , no AIDS and nadir CD4 200-499 cells/ $\mu \mathrm{L}$ (or CD4\% 14-<29); Stage 3, AIDS or nadir CD4 0-199 cells/ $\mu \mathrm{L}$ (or CD4\% $<14$ ).

${ }^{\mathrm{g}}$ Percentages within those who self-reported "currently taking ART".

Among persons that were prescribed ART and initiated ART in the past year, $80.5 \%$ were prescribed a recommended initial regimen, $4 \%$ an alternative initial regimen, $10 \%$ a not-recommended regimen, and $5.4 \%$ other regimens (Table 4). About half (45.7\%) were prescribed the regimen combination of EFV/TDF/XTC. Persons prescribed EFV/TDF/XTC also had the highest dose adherence (88.8\%). Among the recommended initial regimens, patients prescribed RAL/TDF/XTC had the highest recent viral suppression (80.6\%).

Table 4. Most recently prescribed antiretroviral regimens preceding interview of HIV-infected adults with ARV initiated within past year and receiving medical care in the United States, Medical Monitoring Project, 2009-2012.

\begin{tabular}{|c|c|c|c|c|}
\hline DHHS Guideline-Specified ARV Regimen as of December 2014 & $\mathbf{n}$ & $\begin{array}{c}\text { Prescribed ARV Regimen } \\
\text { Col\% (CI) } \\
\end{array}$ & \begin{tabular}{|c|} 
Recent VL Suppressed \\
Row\% (CI) \\
\end{tabular} & \begin{tabular}{|c|} 
Dose Adherent \\
Row\% (CI) \\
\end{tabular} \\
\hline Recommended Initial Regimens & 680 & $80.5(77.6-83.5)$ & $70.4(66.9-74.0)$ & $85.4(82.9-88.0)$ \\
\hline EFV/TDF/XTC & 375 & $45.7(41.7-49.6)$ & $71.2(66.8-75.5)$ & $88.8(85.5-92.0)$ \\
\hline $\mathrm{ATVr} / \mathrm{TDF} / \mathrm{XTC}$ & 121 & $13.8(11.4-16.3)$ & $67.7(58.7-76.7)$ & $80.5(73.1-87.9)$ \\
\hline DRVr/TDF/XTC & 64 & $7.1(5.4-8.9)$ & $66.5(52.6-80.3)$ & $85.0(76.4-93.6)$ \\
\hline $\mathrm{RAL} / \mathrm{TDF} / \mathrm{XTC}$ & 57 & $6.3(4.4-8.3)$ & $80.6(69.0-92.2)$ & $85.2(74.5-96.0)$ \\
\hline $\mathrm{EFV} / \mathrm{ABC} / \mathrm{XTC}$ & $8^{\#}$ & & & \\
\hline $\mathrm{ATVr} / \mathrm{ABC} / \mathrm{XTC}$ & $\sigma^{\#}$ & & & \\
\hline EVG/TDF/FTC & $4^{*}$ & & & \\
\hline RPV/ TDF/XTC & 45 & $5.5(3.3-7.8)$ & $60.2(46.2-74.3)$ & $75.8(63.4-88.2)$ \\
\hline Alternative initial regimens & 31 & $4.0(2.4-5.7)$ & $52.7(34.1-71.3)$ & $62.1(41.5-82.7)$ \\
\hline $\mathrm{DRVr}+\mathrm{ABC} / \mathrm{XTC}$ & $4^{\#}$ & & & \\
\hline $\mathrm{LPVr}+\mathrm{ABC} / \mathrm{XTC}$ & 0 & & & \\
\hline $\mathrm{LPVr}+\mathrm{TDF} / \mathrm{XTC}$ & 23 & $3.2(1.7-4.6)$ & & \\
\hline $\mathrm{RAL}+\mathrm{ABC} / \mathrm{XTC}$ & $4^{*}$ & & & \\
\hline NOT recommended as initial regimens & 93 & $10.0(7.9-12.1)$ & $62.5(49.2-75.7)$ & $78.8(66.7-90.8)$ \\
\hline Other regimens & 50 & $5.4(3.9-6.9)$ & $65.0(50.9-79.0)$ & $83.5(72.8-94.2)$ \\
\hline Total & 854 & 100 & $68.6(65.1-72.2)$ & $83.7(81.4-86.1)$ \\
\hline
\end{tabular}

Abbreviations: n, sample size; \%, weighted percent; CI, 95\% confidence interval; VL, HIV viral load; DHHS, U.S. Department of Health and Human Services.

ARV abbreviations: 3TC, lamivudine; ABC, abacavir; APV, amprenavir; ATV, atazanavir; d4T, stavudine; ddC, zalcitabine; ddI, didanosine; DLV, delavirdine; DRV, darunavir; DTG, dolutegravir; EFV, efavirenz; ETV, etravirine; EVG, elvitegravir; FOS-APV, fosamprenavir; FTC, emtricitabine; IDV, indinavir; LPV, lopinavir; MVC, maraviroc; NFV, nelfinavir; NVP, nevirapine; RAL, raltegravir; r, ritonavir-boosted; RPV, rilpivirine; RTV, 
ritonavir; SQV, saquinavir; T-20, enfuvirtide; TDF, tenofovir; TPV, tipranavir; XTC, lamivudine or emtricitabine; ZDV, zidovudine.

${ }^{\#}$ Population estimate was not provided because coefficient of variance exceeded $30 \%$.

Our sensitivity analysis compared those who had an ART initiation date to those who did not have one. Patients who had missing initiation dates were older ( $>50$ years), non- Hispanic black, had more than a high school education, lived at or below poverty level, experienced homelessness, and had been diagnosed more than 10 years ago compared to those who reported ART initiation dates (Supplemental Table 3).

\section{DISCUSSION}

In this large, geographically diverse, nationally representative sample of HIV-infected persons in care, $91.8 \%$ were prescribed ART with the percentage prescribed ART significantly higher in 2012 compared to 2009. Overall, persons prescribed ART regimens were more likely to be older and later in the course of their disease (e.g. longer history of diagnosed with HIV, more likely to have AIDS). Among persons prescribed ART, over half were prescribed a recommended initial regimen, while within those who initiated ART in the past year, over $80 \%$ were prescribed a recommended initial regimen. $\mathrm{EFV} / \mathrm{TDF} / \mathrm{XTC}$ was the most commonly prescribed regimen.

Although over $80 \%$ of persons who initiated ART in the past year were prescribed a recommended initial regimen, only $52 \%$ of all persons were prescribed a recommended initial regimen. Because ARV prescribing should be driven by clinician judgment and risk-benefit analysis of numerous ARV options, it is reassuring that relatively few persons were prescribed ARV regimens that have inferior efficacy or tolerability (e.g. ABC/3TC/ZDV, stavudine + others, nelfinavir + others) [1]. It is possible that most persons prescribed not-recommended regimens might be on second or third-line regimens that use second-line ARVs given limited treatment options due to resistance. In addition, this analysis highlights the reliance by providers on certain ARVs that are key components of most regimens (e.g. $72.5 \%$ of patients were on TDF; $66.0 \%$ on FTC; $44.5 \%$ on ritonavir). Some of these ARVs have known side effects (e.g. risk for renal dysfunction with TDF [20], drug-drug interactions with ritonavir [21]) and thus put a large percentage of the entire HIV-infected population at risk for developing these complications.

Although substantial data exist from randomized clinical trials on the efficacy and safety of particular ARV regimens, observational data from real-world clinical settings can be used to assess the real-world effectiveness and tolerability of ARV regimens, as persons who participate in clinical trials are often different than those in the general HIV-infected population (e.g. injection drug users, homeless persons, and racial and ethnic minorities are underrepresented in clinical trials) [22 - 25]. Data from clinical trials suggest efficacy approaching $90 \%$ for most recommended regimens, but we found that overall only $79.5 \%$ achieved recent viral suppression and $65.5 \%$ achieved durable viral suppression. This gap between ARV regimen efficacy noted in clinical trials and real-world effectiveness is important for a few reasons. First, it helps informs national targets for achieving viral suppression and thus helps to estimate the expected drop-off between ART prescription and viral suppression at the population-level. For example, in this analysis, as in other U.S.-based studies [26 - 28], fewer than 90\% of patients on ART achieved viral suppression, thus suggesting that international targets such as $90-90-90$, which state that $90 \%$ of persons prescribed ART should achieve viral suppression, might be difficult to attain [29]. Second, as shown in this analysis and by others, certain sociodemographic (e.g. younger age, homeless), behavioral (e.g. injection drug use), and clinical (e.g. depression) factors were associated with decreased prevalence of viral suppression [30 - 36] and, as shown previously, persons with these characteristics are less likely to participate in HIV clinical trials [22 - 25]. Thus, population-level differences between all HIV-infected persons and those who participate in clinical trials might contribute to reduced real-world ART effectiveness compared to efficacy seen in clinical trials. A better understanding of the key factors associated with the gap between efficacy in clinical trials and real-world effectiveness could be used to design interventions to improve ART effectiveness.

Among top 5 most frequently prescribed ARV regimens, persons prescribed EFV/TDF/XTC were highest for recent viral suppression, durable viral suppression, fully dose adherent in past 72 hours, and lowest in reporting side effects. As newer recommended regimens are adopted in clinical practice (e.g. integrase strand transfer inhibitor-based regimens), additional analyses of population-level data on viral suppression and dose adherence should be conducted to confirm that benefits noted in clinical trials translate into real-world performance.

This study has a number of limitations. First, MMP is an annual, cross sectional survey and thus participants were not randomly allocated to regimen types. There may be possible survivor or selection bias among our patients, as the median time since ART initiation was nearly 10 years, and may have additional factors that contributed to virologic control and overall healthcare. Second, data on prior treatment history and ARV resistance were unavailable and thus, 
we could not restrict this analysis only to patients who were ARV naïve and did not have transmitted ARV resistance. As has been shown previously, patients on second and third line regimens are likely to have lower levels of viral suppression, lower adherence and more side effects [1,37]. Moreover, we did not have information on all factors that might inform a clinician's decision to prescribe an initial regimen, such as HLAB5701 testing or initial viral load. Third, ART initiation dates were self-reported and thus is subjected to recall bias if participants were diagnosed a long time ago. In our study, the majority (65\%) of those with missing ART entry dates were those diagnosed more than 10 years ago. Fourth, dose adherence and side effect data were self-reported and were not collected using a systematic checklist such as might be done in a safety assessment in a clinical trial. In addition, symptom severity was not assessed. Fifth, DHHS guidelines change over time and thus some regimens that were recommended in 2009 (e.g. $\mathrm{LPVr} / \mathrm{ZDV} / 3 \mathrm{TC}$ ) might no longer be on the recommended list in 2014. This is a major limitation in using this data to assess adherence to a single standard of care. However, our intention was to describe prescribing patterns and ART use rather than assessing adherence to a single set of guidelines. Moreover, this analysis includes data collected through May 2013 and thus does not include much information on newer ARV regimens, such as those containing elvitegravir or dolutegravir. Additionally, our study showed that ritonavir is the most commonly prescribed protease inhibitor (44\%). In practice, ritonavir was mainly used as a pharmacokinetic enhancer and co-prescribed with other protease inhibitors such as atazanavir, lopinavir or darunavir, rather than a standalone ART. However, we do not have ritonavir dosing information in the current MMP dataset; thus our analysis cannot address this issue. Finally, our patient response rates ranged from $49 \%$ to $55 \%$, although our use of population-based sampling methods and weighting adjustments for nonresponse should reduce bias [38], and the MMP population is demographically similar to all HIV-diagnosed persons in the United States [39]. Furthermore, our study participants are limited to HIV-positive persons who are known to be in medical care. We have no information about HIV-positive persons who are not in care or who have not been diagnosed with HIV. While important, the results of this analysis should be interpreted with caution when discussing all HIV-positive persons.

\section{CONCLUSION}

In conclusion, over $90 \%$ of HIV-infected adults receiving medical care in the United States were prescribed ART. Of those prescribed ART, just over half were prescribed recommended initial regimens, which were found to be effective and tolerable. HIV providers should continue to prescribe ART according to established guidelines [1]. Given that new ARV regimens are being introduced, monitoring of ARV use at the population-level should be continued to provide ongoing assessments of ART effectiveness and tolerability in the United States.

\section{FUNDING INFORMATION}

Funding for the Medical Monitoring Project is provided by a cooperative agreement (PS09-937) from the Centers for Disease Control and Prevention.

\section{DISCLAIMER}

The findings and conclusions in this article are those of the authors and do not necessarily represent the official position of the Centers for Disease Control and Prevention or the U.S. Department of Health and Human Services.

\section{ETHICS APPROVAL AND CONSENT TO PARTICIPATE}

Not applicable.

\section{HUMAN AND ANIMAL RIGHTS}

No Animals/Humans were used for studies that are base of this research.

\section{CONSENT FOR PUBLICATION}

Not applicable.

\section{CONFLICT OF INTEREST}

The authors declare no conflict of interest, financial or otherwise. 


\section{ACKNOWLEDGEMENTS}

We thank participating MMP patients, facilities, project areas, and Provider and Community Advisory Board members. We also acknowledge the contributions of the Clinical Outcomes Team and Behavioral and Clinical Surveillance Branch at CDC and the MMP 2009-2012 project areas. (http://www.cdc.gov/hiv/statistics/ systems/mmp/resources.html).

\section{REFERENCES}

[1] Panel on Antiretroviral Guidelines for Adults and Adolescents. Guidelines for the use of antiretroviral agents in HIV-1-infected adults and adolescents 2014. Available from http://aidsinfo.nih.gov/contentfiles/lvguidelines/AdultandAdolescentGL.pdf

[2] Food and Drug Administration. Antiretroviral drugs used in the treatment of HIV infection 2014. Available from: http://www.fda.gov/forpatients/illness/hivaids/treatment/ucm118915.htm

[3] Cohen CJ, Molina JM, Cassetti I, et al. Week 96 efficacy and safety of rilpivirine in treatment-naive, HIV-1 patients in two Phase III randomized trials. AIDS 2013; 27(6): 939-50.

[http://dx.doi.org/10.1097/QAD.0b013e32835cee6e] [PMID: 23211772]

[4] Daar ES, Tierney C, Fischl MA, et al. Atazanavir plus ritonavir or efavirenz as part of a 3-drug regimen for initial treatment of HIV-1. Ann Intern Med 2011; 154(7): 445-56.

[http://dx.doi.org/10.7326/0003-4819-154-7-201104050-00316] [PMID: 21320923]

[5] Lennox JL, DeJesus E, Lazzarin A, et al. Safety and efficacy of raltegravir-based versus efavirenz-based combination therapy in treatmentnaive patients with HIV-1 infection: A multicentre, double-blind randomised controlled trial. Lancet 2009; 374(9692): 796-806. [http://dx.doi.org/10.1016/S0140-6736(09)60918-1] [PMID: 19647866]

[6] Lennox JL, Landovitz RJ, Ribaudo HJ, et al. Efficacy and tolerability of 3 nonnucleoside reverse transcriptase inhibitor-sparing antiretroviral regimens for treatment-naive volunteers infected with HIV-1: A randomized, controlled equivalence trial. Ann Intern Med 2014; 161(7): 461-71.

[http://dx.doi.org/10.7326/M14-1084] [PMID: 25285539]

[7] Molina JM, Clumeck N, Orkin C, Rimsky LT, Vanveggel S, Stevens M. Week 96 analysis of rilpivirine or efavirenz in HIV-1-infected patients with baseline viral load $\leq 100000$ copies/mL in the pooled ECHO and THRIVE phase 3, randomized, double-blind trials. HIV Med 2014; 15(1): 57-62.

[http://dx.doi.org/10.1111/hiv.12071] [PMID: 23980523]

[8] Rockstroh JK, DeJesus E, Lennox JL, et al. Durable efficacy and safety of raltegravir versus efavirenz when combined with tenofovir/emtricitabine in treatment-naive HIV-1-infected patients: final 5-year results from STARTMRK. J Acquir Immune Defic Syndr 2013; 63(1): 77-85.

[http://dx.doi.org/10.1097/QAI.0b013e31828ace69] [PMID: 23412015]

[9] Smith KY, Tierney C, Mollan K, et al. Outcomes by sex following treatment initiation with atazanavir plus ritonavir or efavirenz with abacavir/lamivudine or tenofovir/emtricitabine. Clin Infect Dis 2014; 58(4): 555-63. [http://dx.doi.org/10.1093/cid/cit747] [PMID: 24253247]

[10] Blair JM, Fagan JL, Frazier EL, et al. Behavioral and clinical characteristics of persons receiving medical care for HIV infection - Medical Monitoring Project, United States, 2009. MMWR Surveill Summ 2014; 63(5): 1-22. [PMID: 24941443]

[11] Frankel MR, McNaghten A, Shapiro MF, et al. A probability sample for monitoring the HIV-infected population in care in the U.S. and in selected states. Open AIDS J 2012; 6: 67-76. [http://dx.doi.org/10.2174/1874613601206010067] [PMID: 23049655]

[12] McNaghten AD, Wolfe MI, Onorato I, et al. Improving the representativeness of behavioral and clinical surveillance for persons with HIV in the United States: the rationale for developing a population-based approach. PLoS One 2007; 2(6): e550. [http://dx.doi.org/10.1371/journal.pone.0000550] [PMID: 17579722]

[13] Centers for Disease Control and Prevention. Data file. Proportion of the estimated number of persons living with HIV in the Medical Monitoring Project compared to National HIV Surveillance Data. Unpublished raw data. October 18, 2018.

[14] Kroenke K, Strine TW, Spitzer RL, Williams JB, Berry JT, Mokdad AH. The PHQ-8 as a measure of current depression in the general population. J Affect Disord 2009; 114(1-3): 163-73.

[http://dx.doi.org/10.1016/j.jad.2008.06.026] [PMID: 18752852]

[15] Kroenke K, Spitzer RL, Williams JB. The PHQ-9: validity of a brief depression severity measure. J Gen Intern Med 2001; 16(9): 606-13. [http://dx.doi.org/10.1046/j.1525-1497.2001.016009606.x] [PMID: 11556941]

[16] Schneider E, Whitmore S, Glynn KM, et al. MMWR Recommendations and reports: Morbidity and mortality weekly report Centers for Disease Control and Prevention. CDC 2008; pp. 1-12.

[17] Rao JNK, Scott AJ. The analysis of categorical data from complex sample surveys: chi-squared tests for goodness-of-fit and independence in two-way tables. J Am Stat Assoc 1981; 76: 221-30. [http://dx.doi.org/10.1080/01621459.1981.10477633] 
[18] Centers for Disease Control and Prevention. Distinguishing public health research and public health non-research 2010. Available from: http://www.cdc.gov/od/science/integrity/docs/cdc-policy-distinguishing-public-health-research-nonresearch.pdf

[19] Services. USDoHaH. Protection of Human Subjects, Title 45 code of Federal Regulations, Part 46. 2009. Available from http://www.hhs.gov/ ohrp/humansubjects/guidance/45cfr46.html

[20] Hall AM, Hendry BM, Nitsch D, Connolly JO. Tenofovir-associated kidney toxicity in HIV-infected patients: a review of the evidence. Am J Kidney Dis 2011; 57(5): 773-80.

[http://dx.doi.org/10.1053/j.ajkd.2011.01.022] [PMID: 21435764]

[21] Josephson F. Drug-drug interactions in the treatment of HIV infection: focus on pharmacokinetic enhancement through CYP3A inhibition. J Intern Med 2010; 268(6): 530-9. [http://dx.doi.org/10.1111/j.1365-2796.2010.02301.x] [PMID: 21073558]

[22] Castillo-Mancilla JR, Cohn SE, Krishnan S, et al. Minorities remain underrepresented in HIV/AIDS research despite access to clinical trials. HIV Clin Trials 2014; 15(1): 14-26. [http://dx.doi.org/10.1310/hct1501-14] [PMID: 24518211]

[23] Gifford AL, Cunningham WE, Heslin KC, et al. Participation in research and access to experimental treatments by HIV-infected patients. N Engl J Med 2002; 346(18): 1373-82.

[http://dx.doi.org/10.1056/NEJMsa011565] [PMID: 11986412]

[24] Gwadz MV, Colon P, Ritchie AS, et al. Increasing and supporting the participation of persons of color living with HIV/AIDS in AIDS clinical trials. Curr HIV/AIDS Rep 2010; 7(4): 194-200. [http://dx.doi.org/10.1007/s11904-010-0055-3] [PMID: 20737252]

[25] Sullivan PS, McNaghten AD, Begley E, Hutchinson A, Cargill VA. Enrollment of racial/ethnic minorities and women with HIV in clinical research studies of HIV medicines. J Natl Med Assoc 2007; 99(3): 242-50. [PMID: 17393948]

[26] Althoff KN, Buchacz K, Hall HI, et al. U.S. trends in antiretroviral therapy use, HIV RNA plasma viral loads, and CD4 T-lymphocyte cell counts among HIV-infected persons, 2000 to 2008. Ann Intern Med 2012; 157(5): 325-35.

[http://dx.doi.org/10.7326/0003-4819-157-5-201209040-00005] [PMID: 22944874]

[27] Dombrowski JC, Kitahata MM, Van Rompaey SE, et al. High levels of antiretroviral use and viral suppression among persons in HIV care in the United States, 2010. J Acquir Immune Defic Syndr 2013; 63(3): 299-306. [http://dx.doi.org/10.1097/QAI.0b013e3182945bc7] [PMID: 23572013]

[28] Moore RD, Keruly JC, Bartlett JG. Improvement in the health of HIV-infected persons in care: reducing disparities. Clin Infect Dis 2012; 55(9): 1242-51. an official publication of the Infectious Diseases Society of America [http://dx.doi.org/10.1093/cid/cis654] [PMID: 23019271]

[29] Joint United Nations Programme on HIV/AIDS (UNAIDS). 90-90-90: An ambitious treatment target to help end the AIDS epidemic 2014. Available from: http://www.unaids.org/sites/default/files/media_asset/90-90-90_en_0.pdf

[30] Adeyemi OM, Livak B, McLoyd P, Smith KY, French AL. Racial/ethnic disparities in engagement in care and viral suppression in a large urban HIV clinic. Clin Infect Dis 2013; 56(10): 1512-4.

[http://dx.doi.org/10.1093/cid/cit063] [PMID: 23386637]

[31] Chander G, Himelhoch S, Moore RD. Substance abuse and psychiatric disorders in HIV-positive patients: epidemiology and impact on antiretroviral therapy. Drugs 2006; 66(6): 769-89. [http://dx.doi.org/10.2165/00003495-200666060-00004] [PMID: 16706551]

[32] Cofrancesco J Jr, Scherzer R, Tien PC, et al. Illicit drug use and HIV treatment outcomes in a US cohort. AIDS 2008; 22(3): 357-65. [http://dx.doi.org/10.1097/QAD.0b013e3282f3cc21] [PMID: 18195562]

[33] Langebeek N, Gisolf EH, Reiss P, et al. Predictors and correlates of adherence to combination antiretroviral therapy (ART) for chronic HIV infection: a meta-analysis. BMC Med 2014; 12 : 142. [http://dx.doi.org/10.1186/s12916-014-0142-1] [PMID: 25145556]

[34] Muthulingam D, Chin J, Hsu L, Scheer S, Schwarcz S. Disparities in engagement in care and viral suppression among persons with HIV. J Acquir Immune Defic Syndr 2013; 63(1): 112-9.

[http://dx.doi.org/10.1097/QAI.0b013e3182894555] [PMID: 23392459]

[35] Protopopescu C, Raffi F, Roux P, et al. Factors associated with non-adherence to long-term highly active antiretroviral therapy: a 10 year follow-up analysis with correction for the bias induced by missing data. J Antimicrob Chemother 2009; 64(3): 599-606. [http://dx.doi.org/10.1093/jac/dkp232] [PMID: 19602563]

[36] Ryscavage P, Anderson EJ, Sutton SH, Reddy S, Taiwo B. Clinical outcomes of adolescents and young adults in adult HIV care. J Acquir Immune Defic Syndr 2011; 58(2): 193-7. [http://dx.doi.org/10.1097/QAI.0B013E31822D7564] [PMID: 21826014]

[37] Napravnik S, Eron JJ, Sterling TR, Juday T, Uy J, Moore RD. Outcomes of second combination antiretroviral therapy regimens among HIVinfected persons in clinical care: a multicenter cohort study. AIDS Res Hum Retroviruses 2013; 29(3): 574-80. [http://dx.doi.org/10.1089/aid.2012.0210] [PMID: 23072322] 
[38] Groves RM. Nonresponse rates and nonresponse bias in household surveys. Public Opin Q 2006; 70: 646-74. [http://dx.doi.org/10.1093/poq/nfl033]

[39] Buchacz K, Frazier EL, Hall HI, et al. A Matter of Perspective: Comparison of the Characteristics of Persons with HIV Infection in the United States from the HIV Outpatient Study, Medical Monitoring Project, and National HIV Surveillance System. Open AIDS J 2015; 9: $123-33$. [http://dx.doi.org/10.2174/1874613601509010123] [PMID: 26793282]

(C) 2018 Tie et al.

This is an open access article distributed under the terms of the Creative Commons Attribution 4.0 International Public License (CC-BY 4.0), a copy of which is available at: https://creativecommons.org/licenses/by/4.0/legalcode. This license permits unrestricted use, distribution, and reproduction in any medium, provided the original author and source are credited. 